\title{
Peptide-PAINT Super-Resolution Imaging Using Transient Coiled Coil Interactions
}

\author{
Alexandra S. Eklund, ${ }^{\#}$ Mahipal Ganji, ${ }^{\#}$ Georgina Gavins, Oliver Seitz, and Ralf Jungmann* \\ Cite This: Nano Lett. 2020, 20, 6732-6737 \\ Read Online
}

ABSTRACT: Super-resolution microscopy is transforming research in the life sciences by enabling the visualization of structures and interactions on the nanoscale. DNA-PAINT is a relatively easy-to-implement single-moleculebased technique, which uses the programmable and transient interaction of dye-labeled oligonucleotides with their complements for super-resolution imaging. However, similar to many imaging approaches, it is still hampered by the subpar performance of labeling probes in terms of their large size and limited labeling efficiency. To overcome this, we here translate the programmability and transient binding nature of DNA-PAINT to coiled coil interactions of short peptides and introduce Peptide-PAINT. We benchmark and optimize its binding kinetics in a single-molecule assay and demonstrate its super-resolution capability using self-assembled DNA origami structures. Peptide-PAINT outperforms classical DNA-PAINT in

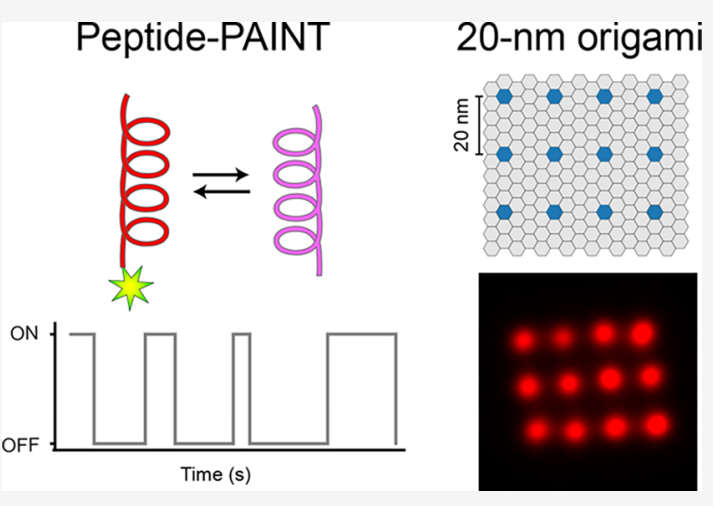
terms of imaging speed and efficiency. Finally, we prove the suitability of Peptide-PAINT for cellular super-resolution imaging by visualizing the microtubule and vimentin network in fixed cells.

KEYWORDS: Super-resolution, coiled coil interactions, transient binding, DNA-PAINT, single-molecule imaging, Peptide-PAINT

$\mathrm{R}$ ecently developed super-resolution fluorescence microscopy approaches allow researchers to characterize cellular structures on the nanoscale. ${ }^{1}$ A widely used super-resolution implementation is single molecule localization microscopy (SMLM), ${ }^{2}$ which uses fluorophore blinking to enable subdiffraction resolution imaging. Blinking can be achieved using photoswitchable proteins or organic dyes as in photoactivated localization microscopy (PALM) ${ }^{3}$ and stochastic optical reconstruction microscopy (STORM) ${ }^{4}$ In points accumulation in nanoscale topography (PAINT), ${ }^{5}$ transient binding interactions between freely diffusing dyes and their targets generate the required blinking pattern, making it easy to implement as photophysical switching of target-bound dye molecules from bright to dark states is not necessary. DNAPAINT, ${ }^{6}$ an adaptation of this concept, uses short dye-labeled DNA oligonucleotides that can transiently, yet sequencespecifically, and repetitively interact with their target-bound complementary strands. Advantages of this approach include better than $5 \mathrm{~nm}$ spatial resolution ${ }^{6,7}$ and high multiplexing capability using DNA-barcoded probes on targets of interest. ${ }^{8}$

One of the main shortcomings of current super-resolution microscopy approaches is due to suboptimal labeling probes like antibodies, which are large in size and have limited labeling efficiency. ${ }^{9}$ While large probes introduce significant linkage artifacts, ${ }^{10,11}$ suboptimal binding efficiency may compromise sensitivity and quantitative detection. ${ }^{12}$ To overcome some of the limitations of antibodies, several different labeling probes have been developed for super-resolution microscopy, including nanobodies, ${ }^{9}$ genetically encoded self-labeling tags (e.g., SNAP and Halo), ${ }^{13,14}$ small protein scaffolds (e.g., affimers or iris probes), ${ }^{15,16}$ and aptamers. ${ }^{17,18}$ However, these methods fall short on delivering all prerequisites for an "ideal" probe, which are (1) site-specific target labeling, (2) smallest label size, (3) highest labeling efficiency, and (4) multiplexing capabilities. Recently, programmable designer peptides generating coiled coil interactions have been identified as potentially ideal probes for fluorescence microscopy with high specificity and small label size. ${ }^{19-23}$ Furthermore, the use of heterodimeric orthogonal coiled coils should allow multiplexing applications. ${ }^{24,25}$ The coiled coil motif involves $\alpha$ helical peptides that wrap around each other and form a superhelix. $^{26}$ An archetypical coiled coil-forming peptide comprises repeats of heptad sequences (hxxhcxc) arranging hydrophobic $(\mathrm{h})$ and charged $(\mathrm{x})$ amino acids residues in $\mathrm{a}$ way that enables mutual recognition of "complementary" peptide strands by means of hydrophobic and ionic interactions ( $x$ could be any amino acid but proline). ${ }^{27,28}$ We envisioned that the use of heterodimeric coiled coils

Received: June 24, 2020

Revised: July 26, 2020

Published: July 27, 2020 

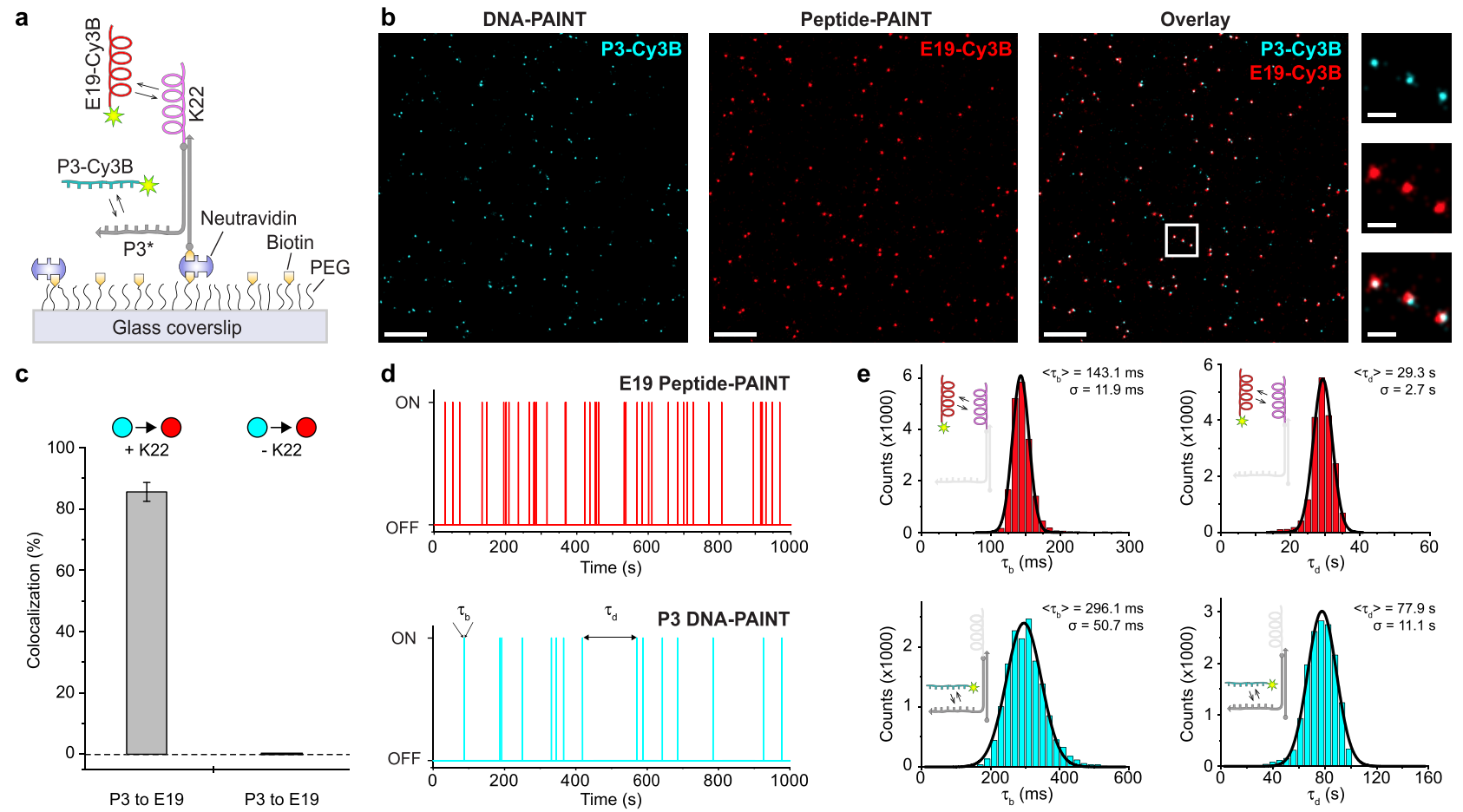

Figure 1. Characterization of transient coiled coil interactions using single-molecule imaging. (a) Schematic diagram depicting the single-molecule imaging assay for characterizing Peptide-PAINT. A peptide coil (K22) is conjugated to a DNA strand (DBCO-S1HP3H, see Table S4) for hybridization to a surface-bound complement. The strand on the coil is furthermore extended with a DNA-PAINT docking sequence for direct visualization. (b) Representative single-molecule localization data resulting from DNA-PAINT (left, cyan), Peptide-PAINT (middle, red), and their overlay (right) of a selected field of view. Zoom-ins highlight colocalization of DNA- and Peptide-PAINT. (c) Colocalization analysis (from DNAto Peptide-PAINT signals) yields $86 \pm 3 \%$ (mean and standard deviation) for the positive control and no colocalization for the negative control, which lacks the K22 peptide $(n>1500)$. (d) Exemplary binding traces showing transient interactions of E19 with surface immobilized K22 and P3 binding to its corresponding docking strand (with highlighted bright and dark times $\tau_{\mathrm{b}}$ and $\left.\tau_{\mathrm{d}}\right)$. (e) $\tau_{\mathrm{b}}$ and $\tau_{\mathrm{d}}$ distributions for E19 ( $\left.n=18938\right)$ and P3 $(n=15544)$ and corresponding Gaussian fits (mean and standard deviation are stated). Scale bars: $300 \mathrm{~nm}$ (b, overview), $50 \mathrm{~nm}(\mathrm{~b}, \mathrm{zoom}$-ins).

enables a design in which one peptide serves as a landing hub for transient interactions with a fluorescence-labeled imager peptide. The mutual interactions between coiled coil peptides are tunable, ${ }^{29}$ potentially making them suitable for PAINTbased super-resolution microscopy.

Here, we rationally redesigned the well-studied, heterodimeric $\mathrm{E} / \mathrm{K}$ coiled coil peptide pair ${ }^{30-32}$ for transient binding to be applicable for PAINT-type super-resolution microscopy and named it Peptide-PAINT. We tuned the coiled coil affinity by varying the length, achieving even faster association kinetics compared to classical DNA-PAINT. The resulting coiled coil pair of approximately 20 amino acids could considerably decrease target-linkage error when genetically fused to a protein of interest, furthermore leading to improved labeling efficiency. We quantitatively characterized the kinetics as well as labeling and imaging efficiency of Peptide-PAINT by singlemolecule imaging. We then used DNA origami structures to demonstrate its suitability for super-resolution microscopy. Finally, we successfully demonstrated Peptide-PAINT in cells by super-resolving the microtubule and vimentin network.

\section{RESULTS AND DISCUSSION}

The PAINT process requires a dynamic equilibrium between bound and unbound states of the fluorescently labeled imager probe. In DNA-PAINT, this is achieved when the transiently formed duplexes have stabilities in the micromolar range $\left(K_{\mathrm{D}}\right.$ $\approx 1 \mu \mathrm{M}$ ). The interaction between the 21 amino acid (aa) long $\mathrm{E}_{3}$ and $\mathrm{K}_{3}$ peptides previously used for protein imaging ${ }^{20-23}$ leads to a coiled coil complex with nanomolar stability $\left(K_{\mathrm{D}} \approx\right.$ $20 \mathrm{nM}$, Figure S1). Aiming for the identification of a suitable weaker affinity, we investigated the length dependence of coiled coil stability (Figure S1). This analysis suggested that complexes comprised of a 22 aa long E peptide and 19 or 18 aa long $\mathrm{K}$ peptides provide stability characteristics $\left(K_{\mathrm{D}} \approx 81 \mathrm{nM}\right.$ or $K_{\mathrm{D}} \approx 1.7 \mu \mathrm{M}$, respectively) suitable for PAINT.

Next, we developed a single-molecule imaging assay to characterize the kinetics of coiled coil interactions between the short peptides. In this assay, the 22 aa long K peptide (K22) served as a docking coil, which was linked to a DNA strand offering an additional docking site for DNA-PAINT. Hybridization with a second biotinylated-DNA strand allowed immobilization on a poly ethylene-glycol (PEG) passivated glass surface via biotin-neutravidin-biotin interactions (Figure 1a). We first located the positions of immobilized molecules via DNA-PAINT imaging of the P3 extension using complementary Cy3B-labeled imager strands (Figure 1b). After a washing step similar to Exchange-PAINT, ${ }^{8}$ we then performed Peptide-PAINT imaging via transient binding of Cy3B-labeled imager peptides (E19) to the immobilized complementary docking peptide (K22). A negatively charged E19-Cy3B imager peptide was used akin to the DNA-PAINT concept, where negatively charged imager strands bind to their targets. This assay enabled us to precisely characterize the kinetic properties and imaging efficiency of transient coiled coil interactions in direct comparison to DNA-PAINT. The multiplexed single-molecule data qualitatively showed a high 

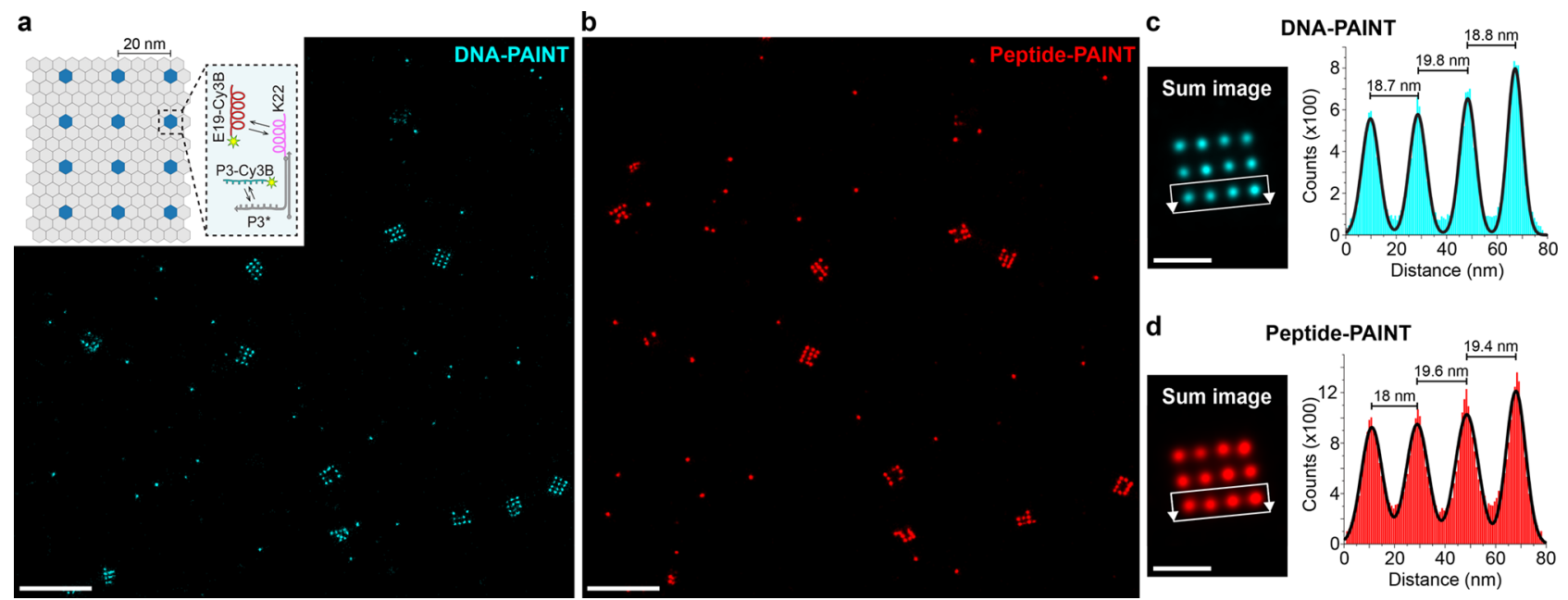

Figure 2. Peptide-PAINT super-resolution imaging of $20 \mathrm{~nm}$ DNA origami grids. (a) DNA-PAINT data (cyan) of $20 \mathrm{~nm}$-grids (inset: schematic representation of $20 \mathrm{~nm}$-grids with single-stranded extensions for DNA- and Peptide-PAINT, reminiscent of single-molecule assay in Figure 1). (b) Peptide-PAINT image (red) of the same region. (c) DNA-PAINT sum image obtained from 100 individual DNA origami (left) and cross-sectional histogram analysis of the localizations in the highlighted region. Gaussian fits yield the designed $20 \mathrm{~nm}$-distances. (d) Corresponding PeptidePAINT analysis. Scale bars: $300 \mathrm{~nm}(\mathrm{a}, \mathrm{b}), 50 \mathrm{~nm}(\mathrm{c}, \mathrm{d})$. The experiment was repeated three times independently with similar results.

degree of colocalization between the immobilized DNA extensions and that of the peptides (Figure 1b, see Figure S2 for whole field of view). To quantify Peptide-PAINT's efficiency, we performed a colocalization analysis from the DNA- to the Peptide-PAINT signal, resulting in $86 \%$ colocalization (Figure 1c). As expected, in the absence of the K22 peptide on the surface-tethered DNA molecules, we observed no Peptide-PAINT signal, indicating that there is no unspecific interaction between the immobilized DNA and the peptide imager (Figure S3).

We observed transient binding kinetics for Peptide-PAINT imaging, similar to DNA-PAINT, indicating that these coiled coil interactions are suitable for super-resolution imaging (Figure 1d, see Table S1 for a binding kinetics overview), yielding comparable localization precisions (3.6 nm for DNAPAINT and $4 \mathrm{~nm}$ for Peptide-PAINT). Intriguingly, time traces of Peptide-PAINT qualitatively show more frequent binding than DNA-PAINT for the same imager concentration. Indeed, statistical analysis of bright and dark times for Peptideand DNA-PAINT demonstrated that the E19 imager peptide manifests faster kinetics than its DNA-PAINT counterpart (Figure 1e), with more than two-times higher association rates (Peptide-PAINT average dark time $=30.0 \pm 0.9$ s, DNAPAINT average dark time $=71.2 \pm 5.5 \mathrm{~s}$ ), ultimately leading to faster image acquisition. ${ }^{33}$ In addition, in an inverse configuration to what is presented in Figure 1a, we showed that E22 docking peptides (immobilized on a surface) can be imaged with K19 imager peptides with similar performance (Figure S4). Peptide-PAINT was also tested in this inverted configuration, in case a positively charged imager peptide (such as $\mathrm{K} 19-\mathrm{Cy} 3 \mathrm{~B}$ ) would be preferred in future studies. In a negative control, where the surface-immobilized DNA molecules lacked the E22 peptide, no detectable binding of K19 imagers to the DNA was observed (Figure S5), once again indicating that the coiled coil interactions are specific and do not interact nonspecifically with DNA.

Similar to the imager-docking strand interactions in DNAPAINT, the binding (or bright) time of coiled coil interactions are also tunable based on the length of the peptide coils. ${ }^{34}$ Indeed, shortening the imager peptide by one amino acid (now called K18), resulted in a reduced bright time $(59.8 \pm 1.6 \mathrm{~ms})$ compared to the 19 amino acid version (K19, $209.5 \pm 2.8 \mathrm{~ms}$ ), with similar dark times (Figure S6). In a negative control lacking E22 peptide, no detectable binding of K18 imagers to the DNA was observed (Figure S7). Due to the relatively short bright times of the K18 imager (potentially advantageous for live cell applications ${ }^{35}$ ), single binding events lead to a reduced number of collectable photons, resulting in suboptimal localization precisions (8.5 $\mathrm{nm}$ for Peptide-PAINT with K18) (Figure S6). Additionally, we note that coiled coil interactions can be tuned ${ }^{29,36}$ by modulating the sequence ${ }^{37}$ and overall charge of the peptide pairs ${ }^{24}$ or the ionic strength of the buffer. ${ }^{38}$ In fact, decreasing the salt concentration by half yields an increased dark time (average dark time for $5 \mathrm{nM}$ E19Cy3B imaging in a $0.5 \times$ PBS buffer is $57.3 \pm 1.6 \mathrm{~s}$ ), whereas increasing the salt concentration results in a reduced dark time, thus increasing acquisition speed (average dark time for $5 \mathrm{nM}$ E19-Cy3B imaging in a $1 \times$ PBS buffer supplemented with 150 $\mathrm{mM} \mathrm{NaCl}$ is $17.2 \mathrm{~s} \pm 3.6 \mathrm{~s}$ ).

Next, we tested the achievable spatial resolution of PeptidePAINT in a more controlled setting using programmable, selfassembled DNA nanostructures. ${ }^{6,39}$ We used $20 \mathrm{~nm}$-grid origami, decorated with DNA-conjugated K22 docking peptides (Figure 2a), reminiscent of the single-molecule assay described above. Using the origami platform, we were able to image $20 \mathrm{~nm}$-grid points with both DNA- and PeptidePAINT with similar spatial resolution (Figure $2 b$ and $c$ ). We used 100 origami structures for averaging (Figure $2 \mathrm{~d}$ and Figure S8) and performed a cross-sectional histogram analysis on Peptide- and DNA-PAINT data, yielding the designed distances for the $20 \mathrm{~nm}$-grid structures. Interestingly, when comparing the normalized number of localizations for both cases, Peptide-PAINT yielded approximately 20\% more localizations, suggesting higher imaging efficiency compared to DNA-PAINT. We note that this difference in efficiency is unlikely to have originated from different accessibility of DNA versus Peptide location, as both were designed with sufficient spacing to the underlying DNA origami structure. In a negative control, where the DNA origami lacked the K22 peptide, no detectable binding of E19 imagers to the DNA nanostructures 

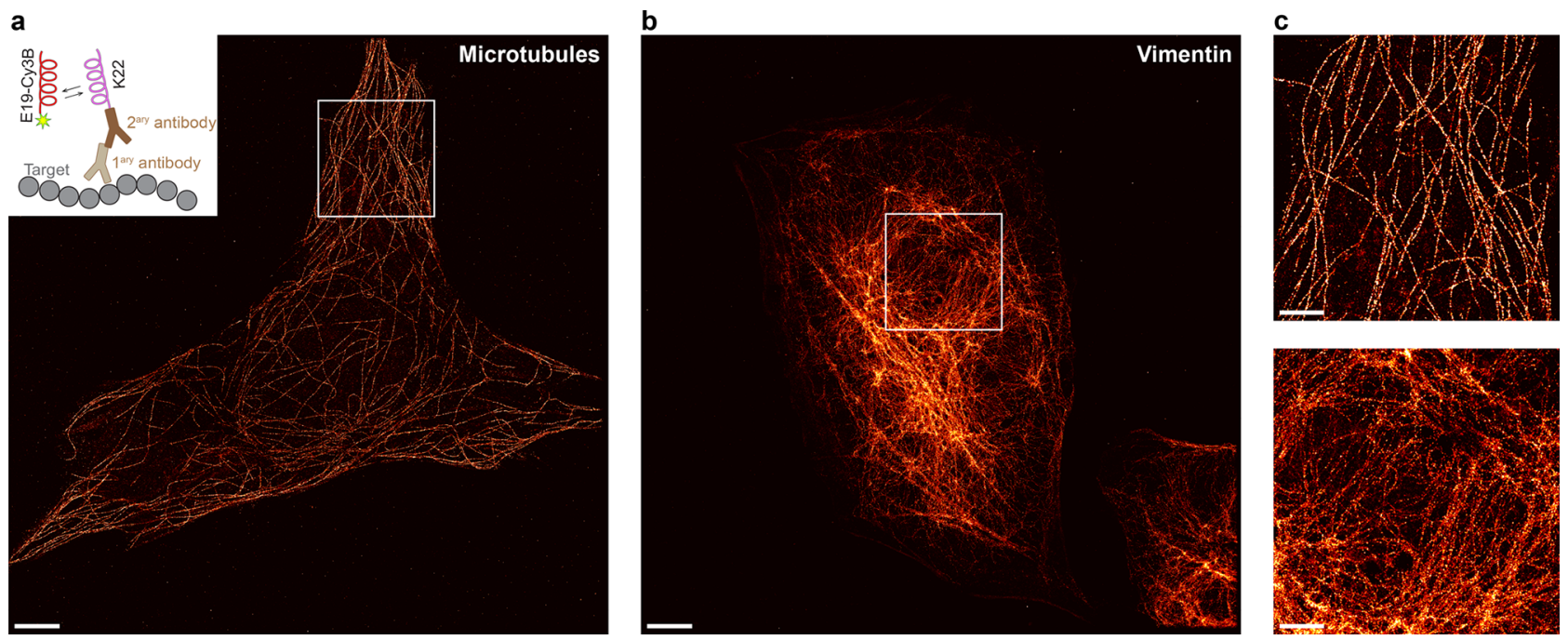

Figure 3. Peptide-PAINT super-resolution imaging of microtubules and vimentin in U2OS cells. (a) Exemplary super-resolved Peptide-PAINT image of microtubules labeled with primary and K22-conjugated secondary antibodies (inset: labeling scheme). (b) Representative super-resolved Peptide-PAINT data of the vimentin network. (c) Zoom-ins of highlighted areas from (a) and (b). Scale bars: $5 \mu \mathrm{m}(\mathrm{a}, \mathrm{b}), 2 \mu \mathrm{m}$ (c). Each experiment was repeated three times independently with similar results.

was observed (Figure S9). We observed similar performance with K19 imager peptides and E22-decorated DNA origami (Figures S10-S12).

We also performed super-resolution imaging with the shorter K18 imager peptide on DNA origami with E22 docking peptides located at each corner of the origami (Figures S13 and S14). We chose these-easier to resolve-fourcornered structures to match the lower localization precision and thus reduced spatial resolution of the K18 imager peptide originating from its shorter binding time (Table S2).

Finally, we sought to demonstrate the ability of PeptidePAINT to image cellular protein structures. In a proof-ofprinciple experiment, we chose microtubules and vimentin as targets because of their unique and recognizable morphology. For this, we labeled U2OS cells with primary antibodies against $\alpha$-tubulin or vimentin, followed by incubation with K22peptide-conjugated secondary antibodies (inset in Figure 3a). Subsequently, Peptide-PAINT imaging was performed with E19 imagers. The reconstructed super-resolution images clearly demonstrate the faithful visualization of the microtubule (Figure 3a) and vimentin filament network (Figure 3b) with high specificity and spatial resolution, similar to previous DNA-PAINT imaging of those targets, ${ }^{10,33,40,41}$ which were not visible in the negative control (Figure S15).

\section{CONCLUSIONS}

In conclusion, we developed Peptide-PAINT as a novel superresolution imaging modality that uses transient interactions of short peptides. We have implemented a single-molecule assay to quantitatively characterize the binding kinetics of these programmable coiled coil interactions and observed that Peptide-PAINT exhibits faster association kinetics compared to classical DNA-PAINT, eventually allowing for faster imaging. We furthermore demonstrated the spatial resolution capabilities of Peptide-PAINT using DNA origami structures. Interestingly, we found that Peptide-PAINT yielded an approximately $20 \%$ improved imaging efficiency compared to DNA-PAINT. In a proof-of-principle experiment, we showed the applicability of Peptide-PAINT for cellular imaging by targeting the microtubule and vimentin network using primary and peptide-conjugated secondary antibodies.

We anticipate exciting future applications for PeptidePAINT based on its improved imaging efficiency and speed compared to classical DNA-PAINT. The speed of PeptidePAINT could be further increased by changing the salt concentration of the buffer ${ }^{33}$ or potentially by increasing the numbers of heptad repeats on the docking strand in combinations with a shorter peptide, similarly to recent speed improvements in DNA-PAINT. ${ }^{41}$ Alternatively, Peptide-PAINT kinetics could be fine-tuned for future applications by varying the sequence of the peptide pairs or the length of the imager peptide. Furthermore, Peptide-PAINT could be extended for multiplexing by adapting orthogonal coiled coil pairs, which have been reported in the literature, ${ }^{24}$ and testing them on our combined single-molecule and DNA origami characterization platform. We anticipate a multiplexing capability of at least six targets from orthogonal coiled coil pairs previously tested in cells. ${ }^{25}$ Due to the small size of the docking peptide, we expect that a genetically encoded peptide tag would be easier to clone and express while causing only minimal distortion to the physiological protein function, in contrast to a larger tag such as GFP (green fluorescent protein). Finally, with the combination of a short imager peptide, we also foresee that Peptide-PAINT will enable highest resolution and labeling efficiency by reducing the linkage error to a minimum and become the preferred labeling strategy for super-resolution microscopy.

\section{ASSOCIATED CONTENT}

\section{SI Supporting Information}

The Supporting Information is available free of charge at https://pubs.acs.org/doi/10.1021/acs.nanolett.0c02620.

Detailed experimental methods and materials; full field of view for the single-molecule experiment; negative controls for single-molecule, origami, and cell experiments; binding kinetics and imaging parameters for DNA-PAINT and Peptide-PAINT; Figures S1-S15 and Tables S1-S8 (PDF) 


\section{AUTHOR INFORMATION}

\section{Corresponding Author}

Ralf Jungmann - Faculty of Physics and Center for Nanoscience, Ludwig Maximilian University, Munich, Germany; Max Planck Institute of Biochemistry, Martinsried, Germany; ๑ orcid.org/ 0000-0003-4607-3312; Email: jungmann@biochem.mpg.de

\section{Authors}

Alexandra S. Eklund - Faculty of Physics and Center for Nanoscience, Ludwig Maximilian University, Munich, Germany; Max Planck Institute of Biochemistry, Martinsried, Germany; @ orcid.org/0000-0003-1047-2100

Mahipal Ganji - Faculty of Physics and Center for Nanoscience, Ludwig Maximilian University, Munich, Germany; Max Planck Institute of Biochemistry, Martinsried, Germany; ๑ orcid.org/ 0000-0001-8176-3322

Georgina Gavins - Institute of Chemistry, Humboldt University, Berlin, Germany

Oliver Seitz - Institute of Chemistry, Humboldt University, Berlin, Germany; @ orcid.org/0000-0003-0611-4810

Complete contact information is available at:

https://pubs.acs.org/10.1021/acs.nanolett.0c02620

\section{Author Contributions}

${ }^{\#}$ These authors contributed equally. A.S.E. conceived and performed experiments, analyzed data, and contributed to the writing of the manuscript. M.G. conceived experiments, analyzed data, wrote the manuscript, and supervised the study. G.G. developed the coiled coil pair and helped with general peptide design. O.S. conceived the study, supervised the study, and edited the manuscript. R.J. conceived and supervised the study, analyzed and interpreted data, and wrote the manuscript. All authors reviewed and approved the manuscript.

\section{Funding}

This work has been supported in part by the German Research Foundation through the Emmy Noether Program (DFG JU 2957/1-1), the SFB1032 (project A11), the European Research Council through an ERC Starting Grant (MolMap, grant agreement number 680241), the Allen Distinguished Investigator Program through The Paul G. Allen Frontiers Group, the Danish National Research Foundation (Centre for Cellular Signal Patterns, DNRF135), the Human Frontier Science Program through a Young Investigator Grant (HFSP RGY0065), and the Max Planck Foundation and the Max Planck Society.

\section{Notes}

The authors declare no competing financial interest.

\section{ACKNOWLEDGMENTS}

We thank Alexander Auer and Philipp Nickels for initial experiments, Thomas Schlichthaerle for fruitful discussions and help with the data analysis, Luis Moroder for helpful discussion, and Sebastian Strauss for help with the cell imaging. We thank Stefan Pettera and Stephan Uebel from the MPIB peptide synthesis service for the production of the peptides and for their advice regarding the peptide modifications. A.S.E. acknowledges support from the International Max Planck Research School for Molecular and Cellular Life Sciences (IMPRS-LS). M.G. acknowledges funding from the European Union's Horizon 2020 research and innovation program under the Marie Skłodowska-Curie grant agreement no. 796606.

\section{ABBREVIATIONS USED}

SMLM, single-molecule localization microscopy; PALM, photoactivated localization microscopy; STORM, stochastic optical reconstruction microscopy; PAINT, points accumulation in nanoscale topography; PEG, poly-ethelene glycol; PBS, phosphate buffer saline; GFP, green fluorescent protein

\section{REFERENCES}

(1) Sahl, S. J.; Hell, S. W.; Jakobs, S. Fluorescence nanoscopy in cell biology. Nat. Rev. Mol. Cell Biol. 2017, 18 (11), 685-701.

(2) Sauer, M.; Heilemann, M. Single-Molecule Localization Microscopy in Eukaryotes. Chem. Rev. 2017, 117 (11), 7478-7509.

(3) Betzig, E.; Patterson, G. H.; Sougrat, R.; Lindwasser, O. W.; Olenych, S.; Bonifacino, J. S.; Davidson, M. W.; Lippincott-Schwartz, J.; Hess, H. F. Imaging intracellular fluorescent proteins at nanometer resolution. Science 2006, 313 (5793), 1642-5.

(4) Rust, M. J.; Bates, M.; Zhuang, X. Sub-diffraction-limit imaging by stochastic optical reconstruction microscopy (STORM). Nat. Methods 2006, 3 (10), 793-5.

(5) Sharonov, A.; Hochstrasser, R. M. Wide-field subdiffraction imaging by accumulated binding of diffusing probes. Proc. Natl. Acad. Sci. U. S. A. 2006, 103 (50), 18911-18916.

(6) Schnitzbauer, J.; Strauss, M. T.; Schlichthaerle, T.; Schueder, F.; Jungmann, R. Super-resolution microscopy with DNA-PAINT. Nat. Protoc. 2017, 12 (6), 1198-1228.

(7) Dai, M.; Jungmann, R.; Yin, P. Optical imaging of individual biomolecules in densely packed clusters. Nat. Nanotechnol. 2016, 11 (9), 798-807.

(8) Jungmann, R.; Avendano, M. S.; Woehrstein, J. B.; Dai, M.; Shih, W. M.; Yin, P. Multiplexed 3D cellular super-resolution imaging with DNA-PAINT and Exchange-PAINT. Nat. Methods 2014, 11 (3), 313-8.

(9) Ries, J.; Kaplan, C.; Platonova, E.; Eghlidi, H.; Ewers, H. A simple, versatile method for GFP-based super-resolution microscopy via nanobodies. Nat. Methods 2012, 9 (6), 582-4.

(10) Schlichthaerle, T.; Ganji, M.; Auer, A.; Kimbu Wade, O.; Jungmann, R. Bacterially Derived Antibody Binders as Small Adapters for DNA-PAINT Microscopy. ChemBioChem 2019, 20 (8), 10321038.

(11) Thevathasan, J. V.; Kahnwald, M.; Cieslinski, K.; Hoess, P.; Peneti, S. K.; Reitberger, M.; Heid, D.; Kasuba, K. C.; Hoerner, S. J.; Li, Y.; Wu, Y. L.; Mund, M.; Matti, U.; Pereira, P. M.; Henriques, R.; Nijmeijer, B.; Kueblbeck, M.; Sabinina, V. J.; Ellenberg, J.; Ries, J. Nuclear pores as versatile reference standards for quantitative superresolution microscopy. Nat. Methods 2019, 16 (10), 1045-1053.

(12) Jungmann, R.; Avendano, M. S.; Dai, M.; Woehrstein, J. B.; Agasti, S. S.; Feiger, Z.; Rodal, A.; Yin, P. Quantitative superresolution imaging with qPAINT. Nat. Methods 2016, 13 (5), 43942.

(13) Keppler, A.; Gendreizig, S.; Gronemeyer, T.; Pick, H.; Vogel, H.; Johnsson, K. A general method for the covalent labeling of fusion proteins with small molecules in vivo. Nat. Biotechnol. 2003, 21 (1), $86-9$.

(14) Los, G. V.; Encell, L. P.; McDougall, M. G.; Hartzell, D. D.; Karassina, N.; Zimprich, C.; Wood, M. G.; Learish, R.; Ohana, R. F.; Urh, M.; Simpson, D.; Mendez, J.; Zimmerman, K.; Otto, P.; Vidugiris, G.; Zhu, J.; Darzins, A.; Klaubert, D. H.; Bulleit, R. F.; Wood, K. V. HaloTag: a novel protein labeling technology for cell imaging and protein analysis. ACS Chem. Biol. 2008, 3 (6), 373-82.

(15) Kiuchi, T.; Higuchi, M.; Takamura, A.; Maruoka, M.; Watanabe, N. Multitarget super-resolution microscopy with highdensity labeling by exchangeable probes. Nat. Methods 2015, 12 (8), 743-746.

(16) Schlichthaerle, T.; Eklund, A. S.; Schueder, F.; Strauss, M. T.; Tiede, C.; Curd, A.; Ries, J.; Peckham, M.; Tomlinson, D. C.; 
Jungmann, R. Site-Specific Labeling of Affimers for DNA-PAINT Microscopy. Angew. Chem., Int. Ed. 2018, 57 (34), 11060-11063.

(17) Opazo, F.; Levy, M.; Byrom, M.; Schafer, C.; Geisler, C.; Groemer, T. W.; Ellington, A. D.; Rizzoli, S. O. Aptamers as potential tools for super-resolution microscopy. Nat. Methods 2012, 9 (10), 938-9.

(18) Strauss, S.; Nickels, P. C.; Strauss, M. T.; Jimenez Sabinina, V.; Ellenberg, J.; Carter, J. D.; Gupta, S.; Janjic, N.; Jungmann, R. Modified aptamers enable quantitative sub-10-nm cellular DNAPAINT imaging. Nat. Methods 2018, 15 (9), 685-688.

(19) Yano, Y.; Yano, A.; Oishi, S.; Sugimoto, Y.; Tsujimoto, G.; Fujii, N.; Matsuzaki, K. Coiled-coil tag-probe system for quick labeling of membrane receptors in living cell. ACS Chem. Biol. 2008, 3 (6), 341-5.

(20) Reinhardt, U.; Lotze, J.; Zernia, S.; Morl, K.; Beck-Sickinger, A. G.; Seitz, O. Peptide-templated acyl transfer: a chemical method for the labeling of membrane proteins on live cells. Angew. Chem., Int. Ed. 2014, 53 (38), 10237-41.

(21) Reinhardt, U.; Lotze, J.; Morl, K.; Beck-Sickinger, A. G.; Seitz, O. Rapid Covalent Fluorescence Labeling of Membrane Proteins on Live Cells via Coiled-Coil Templated Acyl Transfer. Bioconjugate Chem. 2015, 26 (10), 2106-17.

(22) Lotze, J.; Wolf, P.; Reinhardt, U.; Seitz, O.; Morl, K.; BeckSickinger, A. G. Time-Resolved Tracking of Separately Internalized Neuropeptide Y2 Receptors by Two-Color Pulse-Chase. ACS Chem. Biol. 2018, 13 (3), 618-627.

(23) Doh, J. K.; White, J. D.; Zane, H. K.; Chang, Y. H.; Lopez, C. S.; Enns, C. A.; Beatty, K. E. VIPER is a genetically encoded peptide tag for fluorescence and electron microscopy. Proc. Natl. Acad. Sci. U. S. A. 2018, 115 (51), 12961-12966.

(24) Gradisar, H.; Jerala, R. De novo design of orthogonal peptide pairs forming parallel coiled-coil heterodimers. J. Pept. Sci. 2011, 17 (2), 100-6.

(25) Lebar, T.; Lainscek, D.; Merljak, E.; Aupic, J.; Jerala, R. A tunable orthogonal coiled-coil interaction toolbox for engineering mammalian cells. Nat. Chem. Biol. 2020, 16 (5), 513-519.

(26) Crick, F. H. C. The packing of $\alpha$-helices: simple coiled-coils. Acta Crystallogr. 1953, 6 (8-9), 689-697.

(27) McLachlan, A. D.; Stewart, M. The 14-fold periodicity in alphatropomyosin and the interaction with actin. J. Mol. Biol. 1976, 103 (2), 271-98.

(28) McLachlan, A. D. The double helix coiled coil structure of murein lipoprotein from Escherichia coli. J. Mol. Biol. 1978, 121 (4), 493-506.

(29) Thomas, F.; Boyle, A. L.; Burton, A. J.; Woolfson, D. N. A set of de novo designed parallel heterodimeric coiled coils with quantified dissociation constants in the micromolar to sub-nanomolar regime. J. Am. Chem. Soc. 2013, 135 (13), 5161-6.

(30) Chao, H.; Houston, M. E., Jr.; Grothe, S.; Kay, C. M.; O'Connor-McCourt, M.; Irvin, R. T.; Hodges, R. S. Kinetic study on the formation of a de novo designed heterodimeric coiled-coil: use of surface plasmon resonance to monitor the association and dissociation of polypeptide chains. Biochemistry 1996, 35 (37), $12175-12185$.

(31) Litowski, J. R.; Hodges, R. S. Designing heterodimeric twostranded alpha-helical coiled-coils. Effects of hydrophobicity and alpha-helical propensity on protein folding, stability, and specificity. $J$. Biol. Chem. 2002, 277 (40), 37272-9.

(32) Gröger, K.; Gavins, G.; Seitz, O. Strand Displacement in Coiled-Coil Structures: Controlled Induction and Reversal of Proximity. Angew. Chem., Int. Ed. 2017, 56 (45), 14217-14221.

(33) Schueder, F.; Stein, J.; Stehr, F.; Auer, A.; Sperl, B.; Strauss, M. T.; Schwille, P.; Jungmann, R. An order of magnitude faster DNAPAINT imaging by optimized sequence design and buffer conditions. Nat. Methods 2019, 16 (11), 1101-1104.

(34) De Crescenzo, G.; Litowski, J. R.; Hodges, R. S.; O’ConnorMcCourt, M. D. Real-time monitoring of the interactions of twostranded de novo designed coiled-coils: effect of chain length on the kinetic and thermodynamic constants of binding. Biochemistry 2003, 42 (6), 1754-63.

(35) Oi, C.; Gidden, Z.; Holyoake, L.; Kantelberg, O.; Mochrie, S.; Horrocks, M. H.; Regan, L. LIVE-PAINT: Super-Resolution Microscopy Inside Live Cells Using Reversible Peptide-Protein Interactions. bioRxiv 2020, 1.

(36) Wood, C. W.; Woolfson, D. N. CCBuilder 2.0: Powerful and accessible coiled-coil modeling. Protein Sci. 2018, 27 (1), 103-111.

(37) Drobnak, I.; Gradisar, H.; Ljubetic, A.; Merljak, E.; Jerala, R. Modulation of Coiled-Coil Dimer Stability through Surface Residues while Preserving Pairing Specificity. J. Am. Chem. Soc. 2017, 139 (24), 8229-8236.

(38) Kohn, W. D.; Kay, C. M.; Hodges, R. S. Salt effects on protein stability: two-stranded alpha-helical coiled-coils containing inter- or intrahelical ion pairs. J. Mol. Biol. 1997, 267 (4), 1039-52.

(39) Rothemund, P. W. Folding DNA to create nanoscale shapes and patterns. Nature 2006, 440 (7082), 297-302.

(40) Agasti, S. S.; Wang, Y.; Schueder, F.; Sukumar, A.; Jungmann, R.; Yin, P. DNA-barcoded labeling probes for highly multiplexed Exchange-PAINT imaging. Chem. Sci. 2017, 8 (4), 3080-3091.

(41) Strauss, S.; Jungmann, R. Up to 100 -fold speed-up and multiplexing in optimized DNA-PAINT. Nat. Methods 2020, 17, 789-791. 